\title{
Estudio comparativo de dos instrumentos para determinar la ubicación del cenit gingival en dientes anterosuperiores.
}

\author{
Comparative study of two instruments to determine the location of gingival zenith of maxillary anterior \\ tooth.
}

Pamela Flores-Jara ${ }^{1, a}$, Leyla Delgado-Cotrina ${ }^{1, b}$, Jacqueline Webb-Linares ${ }^{1, b}$, Alexis Evangelista-Alva ${ }^{1, b}$.

\section{RESUMEN}

Objetivo: Determinar la ubicación y nivel del cenit gingival de dientes anterosuperiores y comparar ambos parámetros con el uso del calibrador digital y compás de puntas secas según género y hemiarcada. Materiales y Métodos: Se incluyeron modelos de estudio del maxilar superior de 20 estudiantes de la Facultad de Estomatología de la Universidad Peruana Cayetano Heredia con edad promedio de 21,05 años (18-24). Se registró en ellos los parámetros mencionados tanto con calibrador digital como con compás y regla. La comparación de mediciones fue mediante la prueba t de Student, y U de Mann Whitney, además se aplicó el coeficiente de correlación de Pearson. Resultados: El promedio del desplazamiento del cenit gingival usando el calibrador digital en el incisivo central (IC), incisivo lateral (IL) y canino (C) fue de $0,72 \mathrm{~mm}$, $0,36 \mathrm{~mm}$ y $0,11 \mathrm{~mm}$ distal del eje longitudinal del diente respectivamente, el promedio con uso del compás fue de $0,71 \mathrm{~mm}$, $0,39 \mathrm{~mm}$ y $0,11 \mathrm{~mm}$ en el IC, IL y C distal del eje axial respectivamente.La distancia promedio del nivel del cenit gingival con calibrador fue de $0,59 \mathrm{~mm}$ y con compás de $0,63 \mathrm{~mm}$. No se observaron diferencias estadísticamente significativas en ninguno de los casos ( $>0,05)$, se encontró alta correlación entre las mediciones con ambos instrumentos, además no se encontró diferencia estadísticamente significativa en las mediciones por género y hemiarcada. Conclusión: El compás puede ser utilizado como método alternativo para dichas mediciones por ser más accesible y sencillo de usar.

Palabras Clave: ESTÉTICA DENTAL, DIENTE, ENCÍA.

\begin{abstract}
\footnotetext{
Facultad de Estomatología Universidad Peruana Cayetano Heredia. Lima, Perú.

a Cirujano Dentista.
}

b Magister en Estomatología.
\end{abstract}

Objective: To locate the displacement of the gingival zenith and the gingival zenith level of the maxillary anterior teeth and compare both parameters using the digital caliper and the two points divider by gender and quadrants. Materials and Methods: It was included models of the maxillary arc of twenty students of the School of Dentistry of the Peruvian University Cayetano Heredia with an average age of 21.05 years (18-24). In the models were measured the mentioned 
parameters with a digital caliper and with a two points divider and a ruler.The test $t$-Student and the test U-Mann Whitney were used to compare measurements; also the Pearson correlation coefficient was applied. Results: The average of the gingival zenith displacement using the digital caliper was of $0.72 \mathrm{~mm}, 0.36 \mathrm{~mm}$ and $0.11 \mathrm{~mm}$ for the central incisor (CI), lateral incisor (LI) and canine (C) distal to the axis respectively, and using the two points divider the measurementswere of $0.71 \mathrm{~mm}, 0.39 \mathrm{~mm}$ and $0.11 \mathrm{~mm}$ for the CI, LI and C distal to the axisrespectively. The distance of the gingival zenith level was of $0.59 \mathrm{~mm}$ with the caliper while with the two points divider was $0.63 \mathrm{~mm}$. There were no statistically significant difference in any of the cases ( $p>0.05)$, it was found a high correlation between both measurements; also there were no significant difference about gender or quadrant. Conclusion: Two points divider can be used as an alternative measurement instrument for the parameters mentioned since is more accessible and easy to use.

\section{Key Words: DENTAL ESTHETIC, TOOTH, GINGIVA.}

\section{INTRODUCCION}

La posición del tejido gingival alrededor de las piezas dentarias en la zona anterior es un elemento primordial en cuanto a la estética $(1,6)$. Las alteraciones dentales $\mathrm{y} / \mathrm{o}$ gingivales en el sector anterior pueden tener un impacto estético en la sonrisa, lo cual puede influenciar en la autoestima e incluso podría cambiar el juicio de las personas con respecto a quien presente dicha alteración (2). Para restablecer esta alteración es necesario contar con ciertos parámetros que permitan brindar armonía entre las estructuras. Uno de estos parámetros es el cenit gingival (CG), definido como el punto más apical del contorno gingival alrededor de las piezas dentarias $(3,4,5)$. Se ha señalado que la ubicación del $\mathrm{C}$ Gen los dientes anteriores se encuentra hacia distal del eje longitudinal de la pieza dental $(7,8)$. Sin embargo, Rufenacht (9). Sarver (10), Ahmad (11), Moncada y Ángel (12) y Kurtzman y Silverstein (13), concuerdan que en el incisivo lateral (IL) el CGcoincide con el eje longitudinal. A partir del 2008 se conocen valores cuantitativos por mediciones con el uso del calibrador digital en modelos de estudio; Mattos y Santana (5) y Chu y col. (1) señalan que el CGtiene un desplazamiento distal con promedios de distancia en el incisivo central (IC) de1mm, en el IL de $0.4 \mathrm{~mm}$ y en el canino (C) de $0 \mathrm{~mm}$. Žagar y col. (2) en el 2010 refieren rangos de desplazamiento distal que varían de 0,95 a $1,01 \mathrm{~mm}$ en el IC, de 0,61 a $0,70 \mathrm{~mm}$ en el IL y de 0,26 a $0,45 \mathrm{~mm}$ en el C.

Diversos factores influyen en la ubicación del CG como la forma (15), la convexidad de la cara labial (11), el género (15), la inclinación axial (12) y la hemiarcada (3). Dientes redondeados presentan el CG coincidente con el eje longitudinal del diente (14). Asimismo, si la convexidad es marcada hacia distal el CG se ubicará en esa posición (11). Se ha reportado que el desplazamiento del $\mathrm{CG}$ es menor en mujeres que en varones (15). La inclinación axial se observa progresivamente hacia distal desde el punto de vista cervical $(12,14)$, por lo que visualmente el CG se ubicaría de igual forma progresivamente hacia distal, haciéndose más evidente en dientes posteriores. Se ha reportado que entre la hemiarcada derecha e izquierda solo existe variación significativa del CG en el caso del C (7).

Por otro lado, la posición del CG es tomada como referencia para determinar el nivel del cenit gingival (NCG), otro parámetro estético a nivel gingival que es definido como la distancia desde el CG del IL hasta una línea imaginaria denominada línea gingival estética que une el CG del IC y del C. Al respecto Marus (16), refiere que la distancia del NCG varía entre $1 \mathrm{a} 2 \mathrm{~mm}$ por debajo de la línea mencionada, Kurtzman y Silverstein (13), señalan un promedio de $1 \mathrm{~mm}$ yotros estudios afirman de forma general que la posición favorable del NCG es ligeramente por debajo de la línea gingival estética $(11,12,17)$. Mattos y Santana (5), reportaron un valor aproximado de 0,75 $\mathrm{mm}$, Charruel y col. (4) usando un software señalaron un promedio de $0,68 \mathrm{~mm}$, Chu y col. (1)refieren que debe medir aproximadamente $1 \mathrm{~mm}$.

Para la medición de estos parámetros se ha utilizado el calibrador digital $(1,3,15,5)$. Sin embargo, este instrumento no es muy accesible, su costo es mayor y su uso es más complejo. Por otro lado, el compás de puntas secas es un instrumento de uso común en la práctica odontológica diaria, a la vez accesible y con menor costo. El propósito del presente estudio fue determinar la ubicación del CG y NCG, así como comparar ambas ubicaciones con el uso del calibrador digital y del compás de puntas secas según género y hemiarcada. 


\section{MATERIALES Y MÉTODOS}

Se incluyeron 20 individuos (14 mujeres, 6 varones) con un promedio de edad de 21,05 años (18-24 años). Se incluyeron individuos con ausencia de restauraciones, lesiones cariosas o no cariosas en el sector anterosuperior, ausencia de diastemas, dientes alineados sin previo tratamiento ortodóncico y ausencia de enfermedad periodontal. Todos los participantes firmaron un consentimiento informado el cual fue aprobado por el Comité Institucional de Ética de la Universidad Peruana Cayetano Heredia (código 57499).

Se tomó impresiones con alginato Neocollid (Zhermack, Italy) que fueron vaciadas con yeso tipo IV Elite Rock (Zhermack, Italy) de acuerdo a las especificaciones del fabricante. Se realizó la medición en el IC, IL y C de cada hemiarcada. Para determinar la posición de la línea media vertical se ubicó el punto más apical e incisal de la zona de contacto mesial y distal trazando líneas horizontales que unieran ambos puntos. A partir del punto medio de éstas se trazó la línea media vertical. Posteriormente se ubicó el CG y desde de éste se trazó una línea vertical descendiente paralela a la línea media. Finalmente se midió la distancia entre ambas líneas paralelas cuantificando el desplazamiento del CG (Fig. 1). Adicionalmente se trazó la línea gingival estética, línea que une el CG del IC y del C, la medición entre esta línea hasta el cenit del IL determinó el NCG (Fig. 2). Las mediciones se realizaron tanto con un calibrador digital $\mathrm{Z} 22855 \mathrm{~F}$ (Powerfix, London, UK)con precisión de $0.01 \mathrm{~mm}$., como con un compás de puntas secas Dentaurum (GmbH y Co.KG, Germany).

Las aperturas de este último se midieron con una regla milimetrada metálica con precisiones de 0,5 $\mathrm{mm}$. El coeficiente de correlación inter clase para la calibración inter examinador usando el compás fue de 0,98 y usando el calibrador de 0,97 , la calibración intra examinador fue en todos los casos mayor a 0,97 .

El análisis estadístico se realizó mediante la prueba $\mathrm{t}$ de Student para muestras independientes y la prueba U de Mann Whitney (con un nivel de significancia del 95\%), además se aplicó el coeficiente de correlación de Pearson para determinar la correlación de mediciones entre el calibrador y el compás.

\section{RESULTADOS}

El promedio del desplazamiento del CG con uso del calibrador digital en el IC, IL y $\mathrm{C}$ es de $0,72 \mathrm{~mm}, 0,36$ $\mathrm{mm}$ y $0,11 \mathrm{~mm}$ distal del eje longitudinal del diente respectivamente, mientras que los promedios con uso del compás son de $0,71 \mathrm{~mm}$ en el IC, $0,39 \mathrm{~mm}$ en el IL y $0,11 \mathrm{~mm}$ en el $\mathrm{C}$ de igual forma hacia distal del eje axial. Los valores entre ambos instrumentos no presentaron diferencia estadísticamente significativa $(\mathrm{p}>0,05)$ (Tabla 1).

Para el IC el 70\% de las ubicaciones del CGse desplazaron hacia distal del eje longitudinal del diente, para el IL el 55\% mostró un desplazamiento distal mientras que un $42.5 \%$ coincidió con el eje longitudinal del diente, en el caso del canino el 77.5\% de las ubicaciones coincidió con el eje axial (Gráfico $1)$.

Al respecto del NCG usando calibrador digital la media fue de $0,59 \mathrm{~mm}$ y usando compás de $0,63 \mathrm{~mm}$. Dichas mediciones tampoco presentaron diferencia estadísticamente significativa entre sí $(p>0,05)$.

La correlación entre las mediciones con calibrador digital y con compás en relación a la ubicación del CG como a la del NCG fue mayor a 0,95 independientemente del diente evaluado $(p<0,01)$ como se muestra en la Tabla 2.

No se encontró diferencia estadísticamente significativa $(\mathrm{p}>0,05)$ en la evaluación por género $y$ por hemiarcada independientemente del instrumento utilizado.

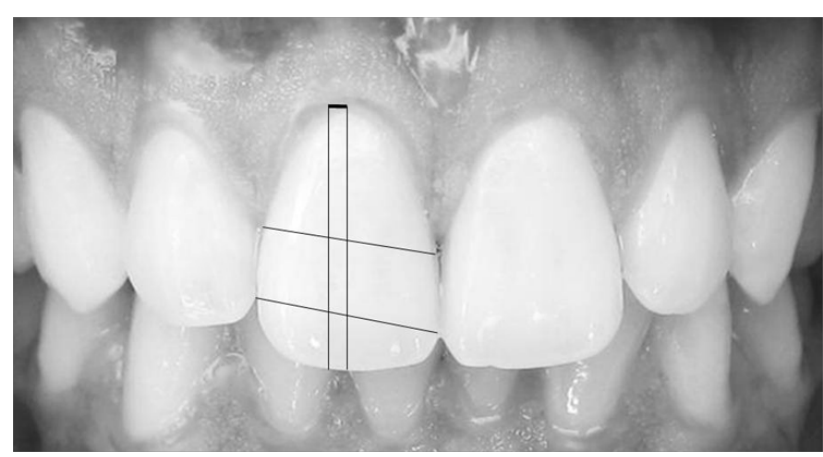

Fig. 1. Esquema utilizado para determinar el desplazamiento del cenit gingival (CG). 


\section{DISCUSIÓN}

Se encontró que los valores promedio del desplazamiento distal del CG al eje axial del diente fueron de $0,72 \mathrm{~mm}$ en el IC, 0,36 mm en el IL y $0,11 \mathrm{~mm}$ en el $\mathrm{C}$ con uso del calibrador digital. Con uso de compás de puntas secas fue de $0,71 \mathrm{~mm}$ en el IC, $0,39 \mathrm{~mm}$ en el IL y $0,11 \mathrm{~mm}$ en el C. Al respecto del NCG se encontró que el promedio es de $0.59 \mathrm{~mm}$ para el calibrador y $0,63 \mathrm{~mm}$ para el compás.

El presente estudio muestra valores similares en cuanto a ubicación, pero en algunos casos menores en cuanto a mediciones comparados con estudios anteriores $(1,5)$. Dicha diferencia puede deberse a la diferente población ya que estudios previos cuentan con población principalmente caucásica y en la presente investigación todos los participantes fueron peruanos.

De acuerdo con los resultados del presente estudio el desplazamiento distal del CG con respecto al eje longitudinal fue en el IC de $70 \%$, en el IL de $55 \%$, mientras que en el $\mathrm{C}$ de $17,5 \%$. Al respecto $\mathrm{Chu}$ y col. (1) publicaron que el $65 \%$ de los IL evaluados en su estudio mostró un desplazamiento distal y para el C el 2,5\% mostró la misma dirección de desplazamiento. Si bien es cierto el promedio de los desplazamientos del presente estudio fueron hacia distal del eje axial, el $30 \%$ de las ubicaciones del CG en el IC, el $42,5 \%$ en el IL y el 77,5\% en el $\mathrm{C}$ se encontraron coincidentes con el eje axial. Se puede deducir entonces que la posición del CG no es ubicada universalmente hacia distal y depende del diente que se evalúe tal como lo refieren Chu y col.(1), Žagar y col. (3), Žagar y col. (15), Charruel y col. (4), Mattos y Santana (5).

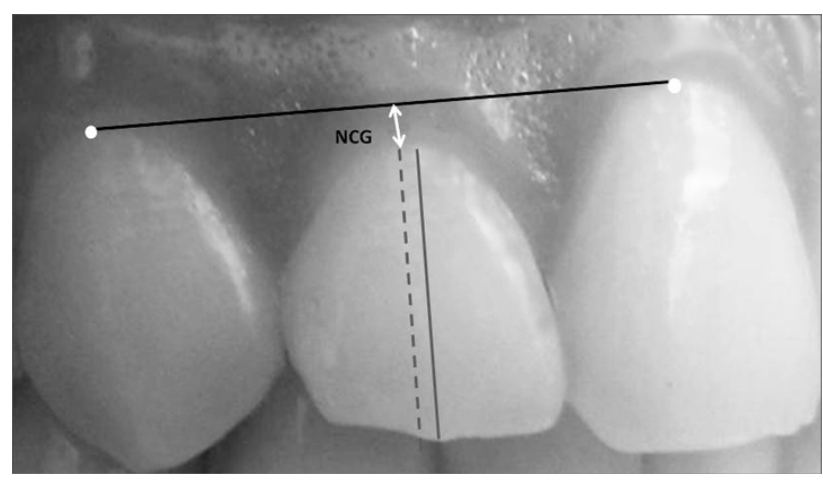

Fig. 2. Esquema utilizado para determinar el nivel del cenit gingival (NCG).
En ninguno de los casos se encontró diferencia estadísticamente significativa entre la medición con el calibrador digital y el compás, sumado a esto los resultados presentan alta correlación entre las mediciones. Estos resultados muestran similitud con la investigación de Williams (18) quien tampoco encontró diferencia significativa entre mediciones realizadas con compás y calibrador, y a la vez encontró alta correlación entre ellas. Entre las posibles causas de la similitud de resultados entre ambos instrumentos se puede mencionar que el compás de puntas secas posee puntas finas que permiten realizar mediciones pequeñas, la regla milimetrada posee precisiones de $0.5 \mathrm{~mm}$ que permite realizar aproximaciones de $0,25 \mathrm{~mm}$ y el calibrador digital permite realizar mediciones de centésimas de milímetro, necesarias quizá para otro tipo de análisis mas no para el presente ya que estas mediciones podrían no ser perceptibles al ojo humano.

No se han reportado estudios anteriores en los cuales se cuantifique la distancia del CG con el uso del compás por lo que sería recomendable continuar con más estudios relacionados.

$\mathrm{Al}$ analizar todos los componentes por hemiarcada se determinó que no existía diferencia significativa entre ambas en ninguno de los casos evaluados, resultados análogos al de Matos y Santana (5) quienes tampoco encontraron diferencia significativa entre ambas hemiarcadas y similares a los de Žagar y col. (15) quienes solo encontraron diferencia significativa para el caso del C. Como se puede apreciar la referencia general viene dada por los promedios tomados a partir del análisis por piezas dentarias agrupadas sin considerar la hemiarcada. La diferencia significativa que encuentra Žagar y col. (15) en cuanto al género con respecto a la ubicación del CG no muestra

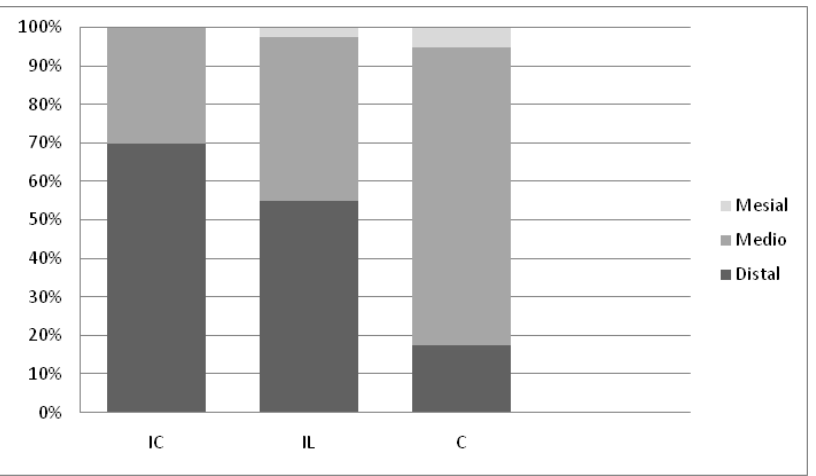

Gráfico 1. Distribución de ubicaciones del cenit gingival en las piezas anterosuperiores. 
Tabla 1. Evaluación del desplazamiento del cenit gingival por pieza anterosuperior.

\begin{tabular}{lcccccc}
\hline INSTRUMENTO & $\mathrm{n}$ & Promedio & Mínimo & Máximo & D.E. & $\mathrm{p}$ \\
\hline Incisivo Central & & & & & & \\
$\quad$ Calibrador & 40 & 0,72 & 0,00 & 2,79 & 0,66 & 0,891 \\
$\quad$ Compás & 40 & 0,71 & 0,00 & 2,75 & 0,63 & \\
$\begin{array}{l}\text { Incisivo Lateral } \\
\quad \text { Calibrador }\end{array}$ & 40 & 0,36 & $-0,91$ & 1,37 & 0,44 & \\
$\quad$ Compás & 40 & 0,39 & $-0,75$ & 1,50 & 0,46 & 0,829 \\
$\quad$ Canino & & & & & & \\
$\quad$ Calibrador & 40 & 0,11 & $-0,63$ & 1,58 & 0,36 & \multirow{2}{*}{0,953} \\
$\quad$ Compás & 40 & 0,11 & $-0,75$ & 1,50 & 0,37 & \\
\hline
\end{tabular}

Valores expresados en milímetros

similitud con el presente estudio ya que en todos los resultados, sean en relación al CG como al NCG no se encontró que exista tal diferencia, probablemente por la incompatibilidad en la población mencionada anteriormente.

Finalmente se deriva que el compás puede ser utilizado como método alternativo en la medición del CG y del NCG además de ser más accesible y sencillo en su uso.

\section{CONCLUSIONES}

- Se observó una tendencia en los dientes anterosuperiores de poseer el CG más coincidente con el eje axial con forme más distal se encuentre el diente en la arcada.

- El desplazamiento promedio del CG con calibrador digital para el IC, IL y C fue de $0,72 \mathrm{~mm}, 0,36$ $\mathrm{mm}$ y $0,11 \mathrm{~mm}$ distal del eje axial respectivamente y con compás fue de $0,71 \mathrm{~mm}, 0,39 \mathrm{~mm}$ y $0,11 \mathrm{~mm}$ para el IC, IL y C respectivamente.

- El promedio del NCG con el uso del calibrador digital fue de $0,59 \mathrm{~mm}$ mientras que con compás fue de $0,63 \mathrm{~mm}$.

- El compás resultó ser tan confiable como el

Tabla 2. Correlación entre la medición con calibrador digital y compás en piezas antero superiores.

\begin{tabular}{lccc}
\hline Medición & $\mathrm{r}$ & $\mathrm{r} 2$ & $\mathrm{p}$ \\
\hline Incisivo central & 0,991 & 0,982 & $<0,001$ \\
Incisivo lateral & 0,988 & 0,976 & $<0,001$ \\
Canino & 0,992 & 0,984 & $<0,001$ \\
$\begin{array}{l}\text { Nivel del cenit } \\
\text { gingival }\end{array}$ & 0,991 & 0,982 & $<0,001$ \\
\hline
\end{tabular}

calibrador digital en cuanto a la medición de desplazamientos del CG y NCG.

\section{CORRESPONDENCIA}

Pamela Flores Jara

Jr. Federico Villarreal 101 Urb. San Agustín II

Comas (Lima 07), Lima, Perú

Correo electrónico: pamela.flores@upch.pe

\section{REFERENCIAS BIBLIOGRÁFICAS}

1. Chu SJ, Tan JHP, Stappert CFJ, Tarnow DP. Gingival zenith positions and levels of the maxillary anterior dentition. J Esthet Restor Dent. 2009;21(2):113-21.

2. Newton JT, Prabhu N, Robinson PG. The impact of dental appearance on the appraisal of personal characteristics. Int J Prosthodont 2003;16(4):42934.

3. Žagar M, Knezovic D, Carek V. A quantitative evaluation of the distal displacement of the gingival zenith in the maxillary anterior teeth. Coll. Antropol. 2010;34(1):225-8.

4. Charruel S, Perez C, Foti B, Camps J, MonnetCorti V. Gingival contour assessment: clinical parameters useful for esthetic diagnosis and treatment. J Periodontol. 2008;79(5):795-801.

5. Mattos CM, Santana RB. A quantitative evaluation of the spatial displacement of the gingival zenith in the maxillary anterior dentition. J Periodontol. 2008;79(10):1880-5.

6. Chu SJ, Tarnow DP, Tan JH, Stappert CF. Papilla proportions in the maxillary anterior dentition. Int J Periodontics Restorative Dent. 2009;29(4):38593. 
7. Wheeler RC. Complete crown form and the periodontium. J Prosthet Dent. 1961;11:722-34.

8. Obradović-Djuricić, K., Kostić, L., Martinović, Z. Gingival and dental parameters in evaluation of esthetic characteristics of fixed restorations. Srpski arhiv za celokupno lekarstvo. 2005;133(3-4):1807.

9. Rufenacht CR. Fundamentos de estética. Sao Paulo. Editorial Quintessence/ Santos; 1990: Pag. 121-7.

10. Sarver DM. Principles of cosmetic dentistry in orthodontics: Part 1. Shape and proportionality of anterior teeth. Am J Orthod Dentofacial Orthop. 2004 Dec;126(6):749-53.

11.Ahmad. Anterior Dental Aesthetics: Gingival Perspective. British Dental J. 2005;199(4): 195 202.

12.Moncada G, Angel P. Parámetros para la evaluación de la estética dentaria antero superior. Rev Dental de Chile. 2008;99(3):29-38.

13.Kurtzman GM, Silverstein LH. Diagnosis and treatment planning for predictable gingival correction of passive eruption. Pract Proced
Aesthet Dent. 2008;20(2):103-8.

14.Blitz N, Steel C, Willhite C. Diagnosis and treatment evaluation in cosmetic dentistry. A guide to accreditation criteria. american academy of cosmetic dentistry.

15.Žagar M, Knezović Zlatarić D. Influence of esthetic dental and facial measurements on the Caucasian patients' satisfaction. J Esthet Restor Dent. 2011;23(1):12-20.

16.Marus R. Treatment planning and smile design using composite resin. Pract Proced Aesthet Dent. 2006;18(4):235-41.

17.Câmara CALP. Estética em Ortodontia: Diagramas de Referências Estéticas Dentárias (DRED) e Faciais (DREF). Rev Dent Press Ortodon Ortop Facial. 2006;11(6):130-56.

18.Williams F. Comparación de los métodos manual con compás de puntas secas y digital (OrtoDig) en la medición de modelos de estudio [Tesis de especialidad]. Lima: Universidad Peruana Cayetano Heredia; 2009.

Recibido : 11-08-2012

Aceptado: 12-11-2012 